\title{
Autonomes Demand Side Management verteilter Energiespeicher
}

\section{Ein Überblick über die Forschungsarbeiten der Forschungsgruppe „Energiesysteme und -komponenten" an der Fachhochschule Vorarlberg}

\author{
P. Kepplinger, B. Fässler, G. Huber, M.A.S.T. Ireshika, K. Rheinberger, M. Preißinger
}

\begin{abstract}
Verbraucherseitige Laststeuerung (Demand Side Management - DSM) wird als ein möglicher Ansatz betrachtet, um die Auswirkungen des Ausbaus von fluktuierenden Erneuerbaren im Stromnetz auszugleichen. Sollen viele verteilte Energiesysteme damit angesprochen werden, stellen zentralistische Ansätze dabei hohe Anforderungen an die Kommunikationsinfrastruktur. Als Alternative wird vielfach eine autonome Laststeuerung (ADSM) mit anreizbasierter Optimierung direkt auf dem Verbrauchergerät betrachtet. Dabei kann die Anreizfunktion mittels unidirektionaler Kommunikation übertragen werden.

Am Forschungszentrum Energie der Fachhochschule Vorarlberg wurden in den letzten Jahren Algorithmen und Prototypen für den Einsatz von ADSM auf verschiedensten verteilten Energiespeichern im elektrischen Stromnetz entwickelt. Dabei werden sowohl thermische Energiespeicher (z. B. HaushaltsWarmwasserspeicher) als auch elektrochemische Speicher (z. B. Batteriespeichersysteme oder Elektroautos) betrachtet. Außerdem werden die Auswirkungen solcher Systeme auf das elektrische Verteilnetz untersucht. Dieser Artikel gibt einen Überblick über die entwickelten Methoden und Ergebnisse aus diesem Forschungsfeld mit dem Ziel, ein weitreichendes Verständnis für die Chancen und Grenzen des ADSM zu schaffen.
\end{abstract}

Schlüsselwörter: Demand Side Management; Lastmanagement; Warmwasserspeicher; Stationäre Batteriespeicher; Elektromobilität

Autonomous demand side management of distributed energy storages: Review of the scientific contributions by the "Energy Systems and Components" research group at the Vorarlberg University of Applied Sciences.

Demand Side Management (DSM) is considered a viable approach to mitigate the effects of the increasing share of strongly volatile energy production by renewables. When many distributed energy storage systems must be integrated, central control approaches have high requirements with regard to communication infrastructure. Autonomous DSM (ADSM) with incentive-driven optimization directly on the device is seen as alternative as it requires only a unidirectional communication to control the distributed flexibility.

At the Research Centre Energy of Vorarlberg University of Applied Sciences, algorithms and prototypes for ADSM of distributed energy storages have been developed over the last years. Both thermal energy storages (e.g. domestic hot water systems) and electro-chemical energy storages (e.g. stationary batteries, electric vehicles) are considered. Moreover, the impact of such systems on distribution grids are investigated. This article gives an overview of the developed methods for ADSM and the results achieved with the aim of creating a broad understanding on the opportunities and limitations of ADSM.

Keywords: demand side management; load management; domestic hot water storage; stationary battery storage; electric vehicles

Eingegangen am 12. September 2019, angenommen am 22. Oktober 2019, online publiziert am 13. Dezember 2019 (c) The Author(s) 2019

\section{Autonomes Demand Side Management}

Durch die Ölpreiskrisen in den 1970er Jahren und die zunehmend erschwerte Planbarkeit des Verbrauchs durch die voranschreitende Elektrifizierung, kam es in den 1980er Jahren zu einem breiten Interesse an einer verbraucherseitigen Laststeuerung. Elektrizitätsversorgungsunternehmen (EVUs) begannen im großen Stil gezielte Maßnahmen zur Beeinflussung von Verbraucherlastgängen zu entwickeln und einzusetzen mit dem Ziel, bestehende Erzeugungskapazitäten möglichst effizient und damit wirtschaftlich zu betreiben [2]. Zeitvariable Tarife oder die Schaltung einzelner Lasten mittels Rundsteuertechnik waren als Konzepte des Demand Side Managements (DSM) bereits entwickelt und klassifiziert. Zudem war die grundsätzliche Fragestellung erstmalig definiert: „Wie ist es möglich, den Lastgang der Verbraucher besser an die Erzeugung anzupassen?" [35].

Obwohl die Problemstellung also seit mehr als 35 Jahren bekannt ist und sich seitdem kaum verändert hat, ist das Interesse am Themengebiet DSM insbesondere in den letzten 15 Jahren stetig gestiegen. Folgende Faktoren können als plausible Treiber genannt werden:
Diese Arbeit wird finanziell unterstützt durch das Bundesministerium für Wissenschaft, Forschung und Wirtschaft und die Nationalstiftung für Forschung, Technologie und Entwicklung.

Kepplinger, Peter, illwerke vkw Stiftungsprofessur für Energieeffizienz, Forschungszentrum Energie, Fachhochschule Vorarlberg, Dornbirn, Österreich (E-Mail: peter.kepplinger@fhv.at), und Josef-Ressel Zentrum für angewandtes wissenschaftliches Rechnen in Energie, Finanzwirtschaft und Logistik, Fachhochschule Vorarlberg, Dornbirn, Österreich; Fässler, Bernhard, illwerke vkw Stiftungsprofessur für Energieeffizienz, Forschungszentrum Energie, Fachhochschule Vorarlberg, Dornbirn, Österreich; Josef-Ressel Zentrum für angewandtes wissenschaftliches Rechnen in Energie, Finanzwirtschaft und Logistik, Fachhochschule Vorarlberg, Dornbirn, Österreich; Faculty of Engineering and Science, University of Agder, Grimstad, Norway; Huber, Gerhard, illwerke vkw Stiftungsprofessur für Energieeffizienz, Forschungszentrum Energie, Fachhochschule Vorarlberg, Dornbirn, Österreich; Ireshika, Muhandiram Arachchige Subodha Tharangi, illwerke vkw Stiftungsprofessur für Energieeffizienz, Forschungszentrum Energie, Fachhochschule Vorarlberg, Dornbirn, Österreich; Josef-Ressel Zentrum für angewandtes wissenschaftliches Rechnen in Energie, Finanzwirtschaft und Logistik, Fachhochschule Vorarlberg, Dornbirn, Österreich; Faculty of Engineering and Science, University of Agder, Grimstad, Norway; Rheinberger, Klaus, illwerke vkw Stiftungsprofessur für Energieeffizienz, Forschungszentrum Energie, Fachhochschule Vorarlberg, Dornbirn, Österreich; Josef-Ressel Zentrum für angewandtes wissenschaftliches Rechnen in Energie, Finanzwirtschaft und Logistik, Fachhochschule Vorarlberg, Dornbirn, Österreich; Preißinger, Markus, illwerke vkw Stiftungsprofessur für Energieeffizienz, Forschungszentrum Energie, Fachhochschule Vorarlberg, Dornbirn, Österreich 


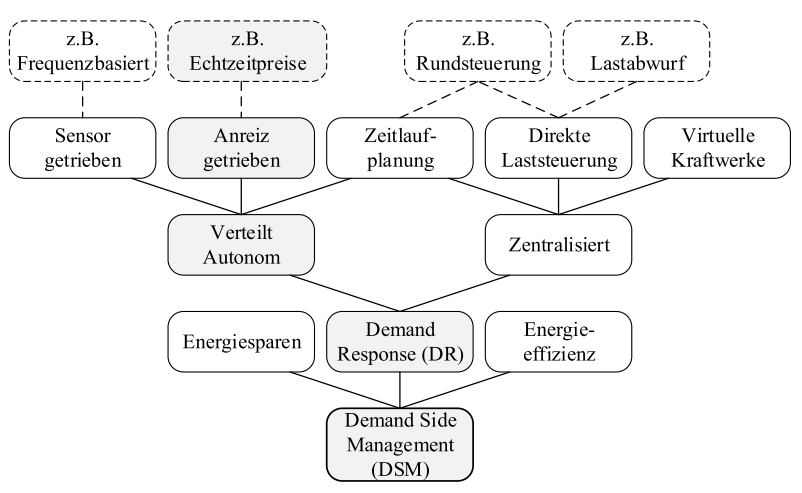

Abb. 1. Ausprägungen des Demand Side Management sowie Verzweigung zum betrachteten autonomen Ansatz (in grau hervorgehoben)

- Integration Erneuerbarer: Europa baut die elektrische Energieversorgung aus erneuerbaren Quellen stetig aus. Stellvertretend sei hier die europäische Roadmap 2050 als politischer Entschluss genannt [7]. Ein Schwerpunkt liegt dabei auf den stark volatilen Erneuerbaren Sonne und Wind. Diese in die elektrische Energieversorgung zu integrieren, stellt jedoch eine große Herausforderung hinsichtlich der Wahrung der Versorgungssicherheit und Netzstabilität dar [29]. DSM wird dabei als mögliche Strategie zur Milderung der auftretenden Probleme diskutiert [27].

- Liberalisierung und Entflechtung des Strommarktes: Die EU Direktiven [3] führten zu einem Markt, der EVUs in neue Geschäftsfelder im Dienstleistungssektor drängt. Hier kann DSM als mögliche Basis für Dienstleistungen, wie z. B. Eigenverbrauchsoptimierung, Lastmanagement auf Gebäude- und Quartiersebene oder Ladelastmanagement von Elektrofahrzeugen dienen.

- Digitalisierung: Die Digitalisierung hat längst Einzug in die elektrische Energieversorgungssysteme gehalten. Potentielle Veränderungen durch digitale Geschäftsmodelle in der Branche werden als immens eingeschätzt [15]. Die derzeit laufende Integration von modernen Verbrauchsmesssystemen (Advanced Metering Infrastructures) durch intelligente Zählersysteme (Smart Meters) in Europa sollen zukünftige Laststeuerungen erleichtern [1].

Die Einteilung von DSM Ansätzen kann vielfältig erfolgen. Eine Möglichkeit nutzte Wayne [35], der DSM Ansätze hinsichtlich des zugrunde liegenden Zwecks kategorisierte: Spitzenlastverschiebung (peak shifting), Schwachlastzeitnutzung (valley filling), Lastverschiebung (load shifting), Verbrauchsreduktion (strategic conservation), strategischer Lastzuwachs (strategic load growth). Abhängig von der Reaktionszeit kann DSM als zweite Möglichkeit in die Märkte Primärregelleistung, Echtzeitpreissysteme, zeitvariable Tarife bis hin zu permanenten Einsparungsmöglichkeiten eingeordnet werden. [26, 34].

Eine dritte Betrachtungsweise ist in Abb. 1 dargestellt und unterteilt zunächst in die drei Bereiche Demand Response, Energieeffizienz und Energieeinsparung. Alle drei Kategorien teilen sich in mögliche technische Umsetzungen auf, wobei in diesem Artikel besonders auf den Bereich Demand Response eingegangen werden soll. ${ }^{1}$

${ }^{1}$ Hier ist zu beachten, dass in der technischen Literatur nicht immer klar zwischen Demand Response (DR) und Demand Side Management (DSM) unterschieden wird; diese Begriffe sind teils historisch unabhängig voneinander in der jeweiligen Spezialisierung gewachsen (Elektrotechnik und Energiewirtschaft).

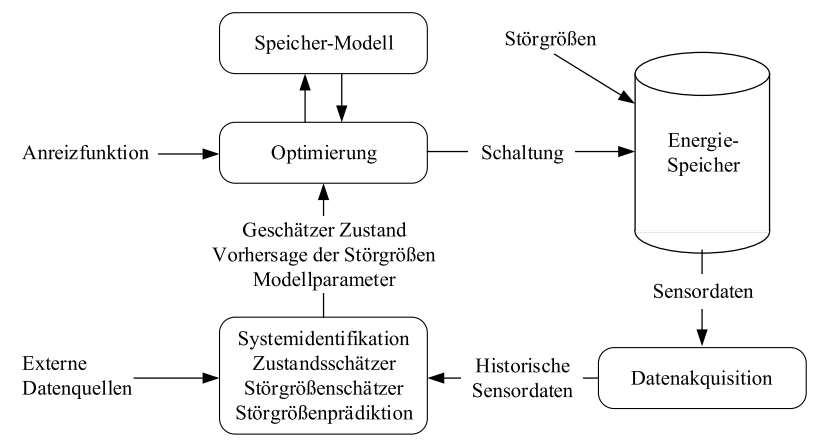

Abb. 2. Autonomes Demand Side Management nach [18]

Der Bereich Demand Response (DR) kann technisch unterschiedlich ausgeprägt sein, je nachdem ob dezentrale oder zentrale Ansätze widergespiegelt werden. Letztere umfassen Virtuelle Kraftwerke und andere direkte Laststeuerungsansätze. Dabei werden an einer zentralen Instanz Entscheidungen über die Schaltzustände vieler Verbraucher getroffen, wobei teilweise Aggregationen und Generalisierungen verwendet werden. Dezentrale Ansätze hingegen räumen den einzelnen Verbrauchern die letztendliche Entscheidung ein, ihre Lasten zu schalten.

Eine weitere Möglichkeit des DR ergab sich durch die Einführung und Weiterentwicklung von kurzfristigen Terminmärkten, wie den heute existierenden 1/4 Stunden Day-Ahead und Intraday Spotmärkten in Europa. Diese führten zu einer starken Diskussion über preisgetriebene Ansätze für die intelligente Steuerung "kleiner" Verbraucher $[25,30]$. In einer verallgemeinerten Form wird ADSM als die Methode bezeichnet, die Verbraucher durch Anreizfunktionen incentivieren. Als typische Anreizfunktionen eignen sich zum Beispiel Echtzeitpreise oder die erwartete Einspeisung Erneuerbarer.

Folgende Vorteile eines dezentralen, autonomen Ansatzes mittels ADSM im Vergleich zu einem zentralen Ansatz für die Steuerung von Energiespeichersystemen sind von besonderer Bedeutung:

- Hohe Datensicherheit durch unidirektionale Kommunikation

- Betriebssicherheit bei Ausfall der zentralen Instanz

- Höhere Modellgenauigkeit durch lokale Datenerfassung

- Reduzierter Kommunikationsaufwand

Dem entgegen stehen folgende Nachteile von ADSM gegenüber zentralen Ansätzen:

- Systemmonitoring nur durch den Endnutzer, nicht durch den Betreiber

- Direktes Lastmanagement über Anreizfunktion nicht möglich

- Abhängigkeiten zu anderen verschiebbaren Lasten (bspw. Energiemanagementsysteme) schwer integrierbar

Die Arbeiten am Forschungszentrum Energie basieren auf dem Konzept, das in Abb. 2 dargestellt ist. Es beruht auf der Idee, anhand einer unidirektional kommunizierten Anreizfunktion die modellprädiktive Regelung des dezentralen Energiespeichers am Gerät vor Ort durchzuführen. Das Energiespeichersystem unterliegt dabei äußeren Störgrößen wie bspw. der Nutzung durch den Verbraucher sowie Umwelteinflüssen. Diese müssen in die optimale Planung miteinbezogen werden. Hierzu werden die in der Datenakquisition gewonnenen Daten lokal verarbeitet um damit den Systemzustand zu charakterisieren. Des Weiteren werden Vorhersagen über den zu erwarteten Einfluss der Störgrößen gemacht, welche zuletzt in die modellbasierte Optimierung des Speicherbetriebs einfließen. Dabei ist stets zu beachten, dass die Implementierung der Routinen möglichst ressourceneffizient im Hinblick auf die Anforderungen an Prozessoren 
und Speicher zu gestalten ist. Nur damit ist gewährleistet, dass die Umsetzung auf einem Embedded System möglich ist [13, 18] und die Ausstattung vieler Endgeräte erfolgen kann - seien es Neugeräte oder idealerweise Endgeräte im Retrofit. Ein Schwerpunkt zeigt sich daher in allen unseren Arbeiten: wir legen Wert auf die Entwicklung einfacher, aber hinreichend genauer Modelle zur Formulierung des Optimierungsproblems und wollen diese auf reduzierter Hardware einsetzbar gestalten.

\section{Aktuelle Forschungsarbeiten}

Im Folgenden wird ein Überblick über zwei abgeschlossene [13,

18] und eine laufende Dissertation sowie mehrere Konferenzbeiträge $[17,19,20,31]$ und Journalbeiträge $[8,9,21,22]$ der Autoren gegeben. Die Ergebnisse werden dabei nach ihren spezifischen Schwerpunkten zusammengefasst.

\subsection{Elektrisch betriebene Warmwasserspeicher}

Elektrisch betriebene Warmwasserspeicher (EWWS) in Haushalten stellen mit ihren durchschnittlichen Speicherkapazitäten von etwa $5 \mathrm{kWh}$ und nominalen Leistungen von rund $2,5 \mathrm{~kW}$ ein bereits in der Vergangenheit intensiv für Lastmanagement genutztes Potential dar. So werden diese seit den 1930er Jahren für DSM Programme eingesetzt, seit den 1980ern sind sie flächendeckend in Rundsteuerprogrammen integriert [14]. Der Betrieb des EWWS im sogenannten Nachttarif (NT) Betrieb stellt dabei eine Art Benchmark dar und wird deswegen in weiterer Folge als Basis für Vergleiche und Potentialabschätzungen autonomer DSM Ansätze herangezogen.

Ziel ist es, den Warmwasserspeicher mit einem Heizelement zu möglichst günstigen Zeiten zu heizen, oder allgemein formuliert: wir wollen die Kosten hinsichtlich einer speziellen Anreizfunktion minimieren. Dabei handelt es sich stets um ein Optimierungsproblem mit Nebenbedingungen. Im konkreten Fall des EWWS sind dies eine Komfortbedingung (minimale Entnahmetemperatur) sowie eine technische Bedingung (Maximaltemperatur im Speicher).

Um das Optimierungsproblem zu lösen, ist ein physikalisches Modell des Speichers nötig. Wird die gesamte Speichermasse als homogen über eine Energiebilanz mit mittlerer Temperatur abgebildet, spricht man von einem Einschichtmodell; darauf basierend ergibt sich ein lineares Optimierungsproblem (LP). Wird der Speicher vertikal raumdiskretisiert und somit über mehrere Energiebilanzen das Schichtungsverhalten abgebildet, spricht man von einem Mehrschichtmodell; es ergibt sich ein nicht-linearen Optimierungsproblem (NLP), für dessen Lösung der Rechenaufwand weitaus höher ist als im Fall des LPs.

Die modellprädiktive Steuerung des Speichers benötigt zudem eine genaue Vorhersage des Warmwasserbedarfs des Nutzers. Daher wird der Warmwasserbedarf basierend auf den akquirierten Daten im Vorhinein über den Optimierungszeitraum hinweg vorhergesagt. Hierfür verwenden wir den k-nearest neighbor (kNN) Algorithmus, der trotz des geringen Rechenaufwands sehr robuste Ergebnisse für die adaptive Vorhersage des Warmwasserbedarfs liefert [20].

Im Rahmen mehrerer Simulationsstudien [19-21] wurden Lastverschiebungs-Potentiale der autonomen Steuerung im Vergleich zum derzeitigen Benchmark des NT-Betriebs abgeschätzt. Die Ergebnisse sind in Tab. 1 zusammengefasst, wobei unterschiedliche Betriebsmodi (NT, LP, NLP) untersucht wurden. Zudem wird die perfekte Nutzervorhersage (PP) mit der Vorhersage des Warmwasserbedarfs basierend auf kNN verglichen. Als Anreizfunktion wurden einstündige Day-Ahead Spotmarktpreise der Energy Exchange Austria (EXAA) herangezogen.

Im Vergleich zum Benchmark erzielen alle optimierten Betriebsweisen monetäre Einsparungen. Das Einschichtmodell mit kNN-
Tab. 1. Ein-Jahres-Simulationen für den optimierten Betrieb von elektrischen Warmwasserspeichern im Vergleich zum Nachttarif-Betrieb nach [20]. Kosten bezüglich dem Day-Ahead Markt, Energieverbrauch und Serviceeinbußen. Ergebnisse für die Optimierung basierend auf einem Einschichtmodell (Lineare Optimierung LP) und einem Mehrschichtmodell (Nichtlineare Optimierung NLP) des Speichers, sowohl für das Potential bei perfekter Vorhersage (PP) des Nutzers, als auch bei Nutzung des k-nearest neighbor Ansatzes (kNN)

\begin{tabular}{lllll}
\hline $\begin{array}{l}\text { Betriebs- } \\
\text { Modus }\end{array}$ & $\begin{array}{l}\text { Nutzer- } \\
\text { vorhersage }\end{array}$ & $\begin{array}{l}\text { Kosten } \\
\text { (EUR) }\end{array}$ & $\begin{array}{l}\text { Energie } \\
(\mathrm{MWh})\end{array}$ & $\begin{array}{l}\text { Service- } \\
\text { einbußen } \\
\left(\mathrm{MK}^{2} \mathrm{~s}\right)\end{array}$ \\
\hline NT & - & 46,77 & 1,58 & 21,6 \\
LP & PP & 36,08 & 1,46 & 0,0 \\
LP & KNN & 41,41 & 1,51 & 16,4 \\
NLP & PP & 32,11 & 1,37 & 0,0 \\
NLP & KNN & 39,25 & 1,49 & 15,4 \\
\hline
\end{tabular}

Vorhersage (LP-kNN) erreicht eine Einsparung von $11 \%$, das Mehrschichtmodell mit perfekter Nutzervorhersage (NLP-PP) erreicht mit über $30 \%$ die maximale Einparung. Obwohl die Zielfunktion der Optimierungsroutine die Kostenminimierung widerspiegelt, werden gleichzeitig auch Energieeinsparungen erzielt. Diese Einsparungen liegen im Bereich von ca. 4 \% (LP-kNN) bis zu einem Maximalwert von $13 \%$ (NLP-PP). Als weiterer Parameter werden die ServiceeinbuBen betrachtet. Diese Einbußen beziffern die Verletzung der Komfortbedingung, d.h. die Unterschreitung der Entnahmetemperaturgrenze von $38^{\circ} \mathrm{C}$ und deren Dauer. Im Vergleich zum NT-Betrieb als Benchmark werden die Serviceeinbußen bei kNN-Prognose des Nutzerverhaltens um 24 \% (LP) bzw. um 29 \% (NLP) verringert, der Komfort für die Benutzer kann somit gesteigert werden. Erwartungsgemäß wären die Serviceeinbußen bei perfekter Nutzervorhersage gleich null, d.h. der Komfort wäre maximal. Die Simulationen zeigen somit, dass bei einem Mehrschichtmodell mit kNN-Nutzervorhersage die Energiekosten und der Energiebedarf sinken und sich gleichzeitig der Komfort erhöht.

In weiterer Folge wurden die Simulationsergebnisse in einem Demonstrator validiert. Hierzu wurde ein handelsüblicher Speicher in einem Haushalt nachgerüstet und anhand einer Anreizfunktion gesteuert. Durch die Vorhersage des Verbrauchs wurde der thermische Wirkungsgrad um 6 \% (Verhältnis der genutzten Energie in Form von Warmwasser zu elektrischer Heizenergie) erhöht [22]. Basierend auf den Erkenntnissen dieses ersten Demonstrators wurde im Rahmen des Smart City Rheintal Projekts [6] ein Prototyp entwickelt, der im Anschluss in 15 Haushalten in einem Zeitraum von über 2 Jahren evaluiert wurde. Erste Ergebnisse dieses Feldversuchs zeigen, dass die vorhergegangenen Simulationsergebnisse im Mittel bestätigt werden können. Insbesondere für die häufig vorkommenden überdimensionierten Warmwasserspeicher kann demnach eine erhebliche Energieeinsparung von über 30 \% erzielt werden.

\subsection{Second-Use-Elektrofahrzeugbatterien}

Durch die stetigen technologischen Verbesserungen eignen sich Batterien zunehmend für den Einsatz als Betriebsmittel zur Wahrung der Versorgungssicherheit und Netzstabilität [32]. Batterien sind praktisch wartungsfrei [24], weisen kurze Reaktionszeiten auf [23], sind sehr effizient mit Systemwirkungsgraden von 65-90\% (inklusive Wechselrichter) $[5,28]$ und können in unterschiedlichen GröBenklassen eingesetzt werden. Allerdings sind die Lebenszykluskosten derzeit noch zu hoch. Eine mögliche Kostensenkung könnte der Second-Use von Elektrofahrzeugbatterien darstellen [33]. Durch die 
Abnahme der Reichweite werden diese bereits bei 70-80 \% ihrer Ursprungskapazität ausgetauscht, eignen sich jedoch weiterhin für die stationäre Anwendung. Zudem wird zukünftig zwangsläufig eine erhebliche Menge an wiederverwertbaren Batterien auf dem Markt verfügbar sein, da die Anzahl an EVs weltweit stetig und dynamisch steigt [16].

Um das technisch-wirtschaftliche Potential eines Kleinspeichers aus wiederverwerteten Elektrofahrzeugbatterien für ADSM abzuschätzen, wurde eine Simulationsstudie durchgeführt sowie ein Demonstrator zur Verifizierung umgesetzt. Als Grundlage der Untersuchung diente eine Natrium-Nickelchlorid Hochtemperatur Batterie ( „ZEBRA“ - Zero Emission Battery Research Activities). Diese stammte aus einem der ersten kommerziellen Elektrofahrzeuge, dem norwegischen Think City. Ziel der Untersuchung war der kostenoptimierte Betrieb des Speichers, als Anreizfunktion diente auch hier der österreichische Day-Ahead Spotmarktpreis.

Erste Simulationsergebnisse mit einem nichtlinearen Batteriemodell zeigten vielversprechende Erlöse, jedoch ist der Rechenaufwand für die nichtlineare Optimierung enorm und damit für die Implementierung auf Embedded Systems mit begrenzter Rechenleistung ungeeignet [12]. Um das temperaturabhängige Systemverhalten der Hochtemperaturbatterie abzubilden, umfasst dieses Modell die elektrochemische und thermische Energiebilanz als gekoppeltes System aus zwei nichtlinearen gewöhnlichen Differentialgleichungen. Durch eine Linearisierung des Batteriemodells und einer linearen Optimierungsroutine konnte der Rechenaufwand der erzielten Simulationsergebnisse um den Faktor 50 reduziert werden [8]. Die Qualitätseinbußen lagen dabei bei weniger als $5 \%$. Im Projekt "Smart City Rheintal" [6] wurde der Ansatz mit einem Demonstrator im Feld überprüft. Der Vergleich des Realbetriebs mit den Simulationsergebnissen zeigte einen Modellfehler von lediglich 7,6 \%, obwohl die Charakteristiken des Batteriemanagementsystems, des Wechselrichters und der Batterie selbst im Optimierungsalgorithmus lediglich stark vereinfacht implementiert wurden. Der geringe Modellfehler lässt sich dabei insbesondere durch auftretende Mittelungseffekte bei langen Simulations- und Betriebszeiten des Batteriespeichers erklären. Somit konnte die technische Machbarkeit des autonomen Speichers im Feld nachgewiesen werden. Zudem zeigte sich auf Basis der aktuellen Spotmarktpreise als Anreizfunktion auch ein wirtschaftliches Potenzial: im Realbetrieb konnten über ADSM Gewinne erzielt werden [9].

\subsection{Netzdienliche Batteriespeicher}

Bei der Bewertung der monetären Effekte von Batteriespeichern sollten stets die Wechselwirkungen mit dem elektrischen Verteilnetz berücksichtigt werden. Daher wurden in einer weiteren Simulationsstudie die Auswirkungen von ADSM auf Batteriespeicher im Hinblick auf ihre Netzdienlichkeit untersucht [10]. Zu diesem Zweck wurde ein Modell eines realen Niederspannungsverteilnetzes mit 49 realen Haushaltslasten erstellt. Drei der Haushalte wurden zusätzlich mit einer Fotovoltaikanlage ausgestattet wurden, um die dezentrale Einspeisung abzubilden. Die Analyse des Verteilnetzes erfolgte mittels dreier Parameter: 1) das Verhältnis von Spitzen- zu Durchschnittsleistung (peak-to-average power ratio - PAPR), 2) der Spannungsabfall und 3) die kumulierten Verteilverlusten, jeweils mit und ohne autonom optimierten Batteriespeicher.

In einem ersten Szenario wurde ein zentraler Speicher am Einspeisepunkt hinzugefügt. Im zweiten Szenario wurden drei dezentrale Speicher an den Haushalten mit Fotovoltaikanlage hinzugefügt, die in Summe dieselbe Speicherkapazität und Leistung des zentralen Speichers aufweisen. Weiters wurden unterschiedliche Anreizfunktionen für die autonome Optimierung getestet, um deren Auswir-

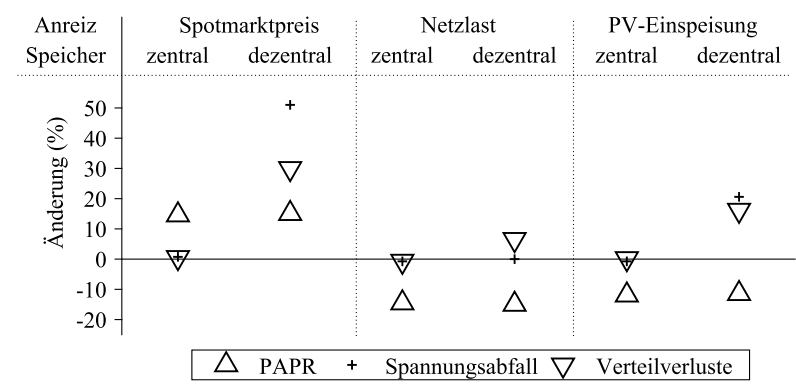

Abb. 3. Relative Änderungen des Spitzen- zu Durchschnittsleistungsverhältniss (PAPR), des Spannungsabfalls und der kumulierten Verteilverluste für alle Anreizfunktionen (Marktpreise, Netzlast, PV-Einspeisung) gegenüber dem Referenzfall ohne Speicher. Betrachtet für einen einzelnen zentralen Speicher und mehrere verteilte Speicher nach [10]

kungen auf das Netz zu untersuchen: 1) Day-Ahead Spotmarktpreise, 2) die zukünftige Netzlast im Verteilnetz und 3) die zukünftige Einspeisung durch Fotovoltaik im Netzgebiet.

Abbildung 3 zeigt dabei, dass Netze insbesondere dann zusätzlich belastet werden, wenn die Anreizfunktion überregionale Märkte wie beispielsweise den Börsenpreis widerspiegelt. Stattdessen ist es empfehlenswert lokal motivierte Anreizfunktionen wie die Netzlast oder die PV-Einspeisung zu verwenden, um mit autonom optimierten Geräten in Niederspannungsverteilnetzen netzdienlich agieren zu können. Dabei bietet sowohl ein einzelner zentraler Speicher als auch mehrere verteilte Speicher Vorteile. Ersteres verbessert die Spannungsqualität und zeigt geringere Verteilverluste, letzteres verbessert das Verhältnis von Spitzen- zu Durchschnittsleistung.

\subsection{Elektrofahrzeuge als Autonome Lasten}

Die erwartete steigende Anzahl an Fahrzeugen mit rein-elektrischen Antrieb (electric vehicle - EV) [16] stellt eine Herausforderung für die bestehenden Niederspannungsnetze dar [4]. Daher ist es wesentlich DSM Ansätze für EVs hinsichtlich ihrer Auswirkungen auf das Verteilnetz zu beurteilen. Während für stationäre Batteriespeicher die optimierte Betriebsplanung im ADSM nur die Randbedingungen des Speichers (Entladetiefe, Kapazität, Wirkungsgrade und Ladeleistung) berücksichtigen muss, stellt beim EV ähnlich zum EWWS die Nutzung eine signifikante Störgröße dar. Dabei sind nicht nur notwendige Mindestladestände zu berücksichtigen, sondern im Fall des EV ist auch eine begrenzte zeitliche Verfügbarkeit für die Ladung vorgegeben. Daher wurde zunächst das entsprechende Optimierungsproblem unter der Annahme der perfekten Vorhersage des Nutzers formuliert [17]. Anschließend wurden die Auswirkungen verschiedener EV-Durchdringungsraten in einem realen Niederspannungsverteilnetz auf Basis von Day-Ahead Spotmarktpreisen untersucht.

Abbildung 4 zeigt exemplarisch einen 24-Stunden-Zyklus bei $5 \%$ Durchdringung und bei $30 \%$ Durchdringung. Für geringe Durchdringungsraten ergibt sich eine Verringerung der Netzlast um ca. $5 \%$, bei höheren Durchdringungsraten führt die Optimierung zu zusätzlichen Lastspitzen von bis zu $50 \%$. Dieses Verhalten ist wiederum auf den Einsatz einer einzigen überregionalen Anreizfunktion zurückzuführen.

\subsection{Design der Anreizfunktion}

Die bisherigen Arbeiten haben gezeigt, dass eine netzdienliche Lastverschiebung nur möglich ist, wenn die Anreizfunktion geschickt gewählt wird. Daher widmen sich die Autoren im Rahmen des Josef Ressel Zentrums für angewandtes wissenschaftliches Rechnen in 

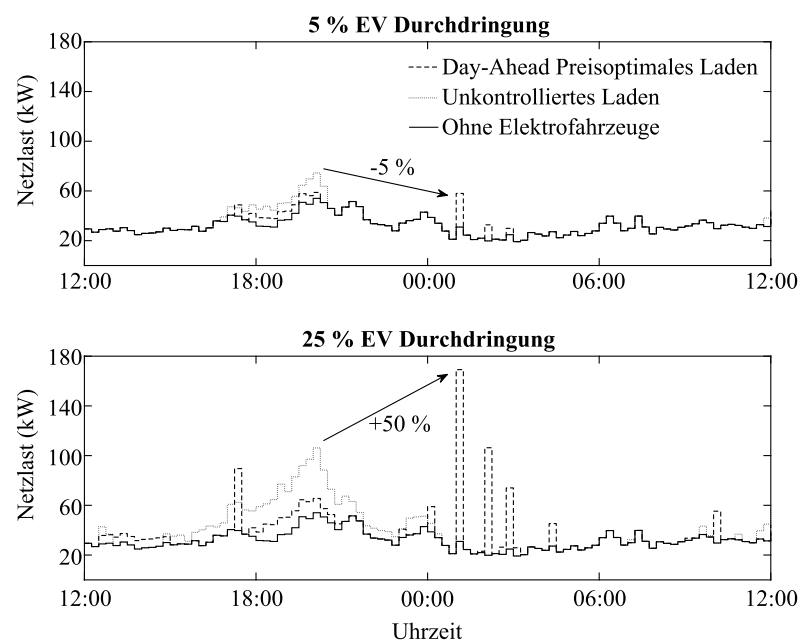

Abb. 4. Auswirkungen des Ladens von Elektrofahrzeugen auf die Netzlast nach [17]

Energie, Finanzwirtschaft und Logistik [11] seit Kurzem auch der Frage nach der optimalen Gestaltung eben dieser Anreizfunktion. Da die Kostenoptimierung durch das Maximumsprinzip bei einer preisgetriebenen Anreizfunktion zu extremen Schaltzuständen und damit zu neuen Lastspitzen führt (vgl. Abb. 4), wurde eine neuartige Anreizfunktion vorgeschlagen, die diese Mängel behebt [31]: der Ziellastgang für den einzelnen Verbraucher. Dieser wird bestmöglich mittels modellprädiktiver Regelung durch den ADSM-fähigen Speicher realisiert. Da der Ansatz eines Lastgangs als Anreizfunktion nach den ersten Ergebnissen als vielversprechend eingestuft werden kann, wenden wir inn bereits in der Weiterentwicklung von netzdienlichen ADSM Ansätzen für EVs an.

\section{Zusammenfassung und Ausblick}

Die vorgestellten Arbeiten zeigen, dass DSM ein sich schnell entwickelndes Feld für die angewandte Forschung ist. Die wichtigsten Erkenntnisse der bisherigen Ergebnisse können wie folgt zusammengefasst werden:

1. ADSM bietet durch seine systemspezifische Implementierung und rasche Skalierbarkeit einen mittelfristig umsetzbaren Weg zur Nutzung von Flexibilitäten.

2. Der optimale Betrieb mittels einer Anreizfunktion kann nicht nur Kosten reduzieren, sondern auch die Effizienz von Technologien steigern und dadurch Energie einsparen.

3. Um die Anforderungen des Verteilnetzes zu erfüllen, müssen DSM Konzepte zukünftig neben überregionalen Märkten auch lokale Begebenheiten durch Erzeugungsanlagen und lokale Netzzustände berücksichtigen.

Die vorgelegten Ergebnisse zeigen Möglichkeiten auf, wie zukunftsfähige und richtungsweisende Lösungen für die angestrebten Entwicklungen hin zu einer nachhaltigen und auch robusten Energieversorgung aussehen könnten.

\section{Danksagung}

Open access funding provided by FH Vorarlberg - University of Applied Sciences. Ein Teil der vorgestellten Arbeiten ist im Rahmen des durch die FFG geförderte Projekt Smart City Rheintal (FFG No. 836088) entstanden. Ein weiterer Teil ist im Rahmen des durch die CDG geförderten Josef Ressel Zentrums für angewandtes wissenschaftliches Rechnen in Energie, Finanzwirtschaft und Logistik entstanden.
Hinweis des Verlags Der Verlag bleibt in Hinblick auf geografische Zuordnungen und Gebietsbezeichnungen in veröffentlichten Karten und Institutsadressen neutral.

Open Access This article is distributed under the terms of the Creative Commons Attribution 4.0 International License (http://creativecommons.org/ licenses/by/4.0/), which permits unrestricted use, distribution, and reproduction in any medium, provided you give appropriate credit to the original author(s) and the source, provide a link to the Creative Commons license, and indicate if changes were made.

Literatur

1. Aghaei, J., Alizadeh, M. I. (2013): Demand response in smart electricity grids equipped with renewable energy sources: a review. Renew. Sustain. Energy Rev., 18, 64-72.

2. Almeida A. T. d., Rosenfeld, A. H. (1988): Demand-side management and electricity end-use efficiency: proceedings of the NATO advanced study institute on demand-side management and electricity end-use efficiency, Povoa de Varzim. NATO ASI series E, applied sciences Povoa de Varzim, Portugal, July 20-31, 1987 (Bd. 149). Dordrecht: Kluwer.

3. Council of European Union (1996): Directive 96/92/ec, 2004/54/ec, 2009/72/ec, 2009.

4. Deb, S., Tammi, K., Kalita, K., Mahanta, P. (2018): Impact of electric vehicle charging station load on distribution network. Energies, 11(1), 178.

5. Dufo-López, R., Bernal-Agustín, J. L. (2015): Techno-economic analysis of gridconnected battery storage. Energy Convers. Manag., 91, 394-404.

6. Eugster, C. (2016): Smart City Rheintal - elements for the emission and energy related spatial development of the polycentric structured rhine-valley. Klima und Energiefonds. Final report.

7. European Commission (2011): Energy roadmap 2050

8. Faessler, B., Kepplinger, P., Petrasch, J. (2017): Decentralized price-driven grid balancing via repurposed electric vehicle batteries. Energy, 118, 446-455.

9. Faessler, B., Kepplinger, P., Petrasch, J. (2019): Field testing of repurposed electric vehicle batteries for price-driven grid balancing. J. Energy Stor., 21, 40-47.

10. Faessler, B., Schuler, M., Preißinger, M., Kepplinger, P. (2017): Battery storage systems as grid-balancing measure in low-voltage distribution grids with distributed generation. Energies, 10(12), 2161

11. Finck, S., Feilhauer, T., Kepplinger, P. (2018): Josef Ressel Zentrum für angewandtes wissenschaftliches Rechnen in Energie, Finanzwirtschaft und Logistik an der Fachhochschule Vorarlberg. In Tagungsband des 12. Forschungsforum der österreichischen Fachhochschulen (FFH) (S. 7). Forschungsforum der österreichischen Fachhochschulen (FFH), 2018

12. Fässler, B, Kepplinger, P., Kolhe, M. L, Petrasch, J. (2015): Decentralized on-site optimization of a battery storage system using one-way communication. In International conference on renewable power generation (RPG 2015) (S. 7).

13. Fäßler, B. (2018): Autonomous optimization of repurposed electric vehicle batteries for grid balancing. Ph.D. thesis, University of Agder, Agder, Norway.

14. Gellings, C. (1985): The concept of demand-side management for electric utilities. Proc. IEEE, 73(10), 1468-1470.

15. IEA (2017): Digitalization and energy. Tech. rep., international energy agency.

16. IEA (Global EV outlook 2019). IEA. Paris, France. Tech. rep, 2019

17. Ireshika, M., Preissinger, M., Kepplinger, P. (2019): Autonomous demand side management of electric vehicles in a distribution grid. In IYCE19, Slovenia: IEEE.

18. Kepplinger, P. (2019): Autonomous demand side management of domestic hot water heaters. Dissertation, University of Innsbruck, Innsbruck, Austria.

19. Kepplinger, P., Huber, G., Amann, P., Rheinberger, K., Petrasch, J. (2013): Active demand side management with domestic hot water heaters using binary integer programming. In e-nova 2013 (Vol. 18, S. 367-376).

20. Kepplinger, P., Huber, G., Petrasch, J. (2014): Demand side management via autonomous control-optimization and unidirectional communication with application to resistive hot water heaters. In e-nova 2014 (Bd. 18, S. 79-86).

21. Kepplinger, P., Huber, G., Petrasch, J. (2015): Autonomous optimal control for demand side management with resistive domestic hot water heaters using linear optimization. Energy Build., 100, 50-55.

22. Kepplinger, P., Huber, G., Petrasch, J. (2016): Field testing of demand side management via autonomous optimal control of a domestic hot water heater. Energy Build. 127.730-735.

23. Khaligh, A., Li, Z. (2010): Battery, ultracapacitor, fuel cell, and hybrid energy storage systems for electric, hybrid electric, fuel cell, and plug-in hybrid electric vehicles: state of the art. IEEE Trans. Veh. Technol., 59(6), 2806-2814.

24. Landgrebe, A. R., Donley, S. W. (1983): Battery storage in residential applications of energy from photovoltaic sources. Appl. Energy, 15(2), 127-137.

25. Mohsenian-Rad, A., Wong, V., Jatskevich, J., Schober, R., Leon-Garcia, A. (2010): Autonomous demand-side management based on game-theoretic energy consumption scheduling for the future smart grid. IEEE Trans. Smart Grid, 1(3), 320-331. 
26. Palensky, P., Dietrich, D., (2011): Demand side management: demand response, intelligent energy systems, and smart loads. IEEE Trans. Ind. Inform., 7(3), 381-388.

27. Paterakis, N. G., Erdinç, O., Catalão, J. P. S. (2017): An overview of demand response: key-elements and international experience. Renew. Sustain. Energy Rev., 69, 871891.

28. Peng, B., Chen, J. (2009): Functional materials with high-efficiency energy storage and conversion for batteries and fuel cells. Coord. Chem. Rev., 253(23-24), 2805-2813.

29. Petinrin, J. O., Shaaban, M. (2016): Impact of renewable generation on voltage control in distribution systems. Renew. Sustain. Energy Rev., 65, 770-783.

30. Ramchurn, S., Vytelingum, P., Rogers, A., Jennings, N. (2011): Agent-based control for decentralised demand side management in the smart grid.

\section{Autoren}

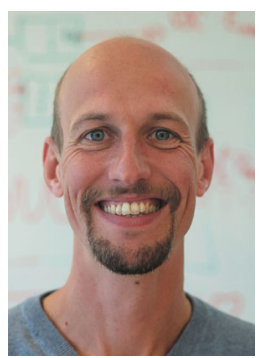

\section{Peter Kepplinger}

schloss 2012 das Studium der Angewandten Mathematik an der Universität Wien ab. Parallel dazu war er von 2008 bis 2012 als Software Entwickler, Applikationsanalyst und Technical Writer tätig. Seit 2013 wissenschaftlicher Mitarbeiter am Forschungszentrum Energie der Fachhochschule Vorarlberg, schloss er 2019 seine Dissertation an der Universität Innsbruck zum Thema „Autonomes Demand Side Management von Elektrisch-Betriebenen Warmwasserspeichern" ab. Seit 2017 ist Peter Kepplinger Leiter der Forschungsgruppe „Energiesysteme und -komponenten". Er und sein Team sind seit 2013 in diversen Forschungsprojekten in Kooperation mit öffentlichen Einrichtungen, privaten Unternehmen und anderen Hochschulen im Bereich des Lastmanagements aktiv. Stellvertretend sei hier das FFG-geförderte Projekt SmartCity Rheintal, sowie das Josef-Ressel Zentrum für Angewandtes Wissenschaftliches Rechnen in Energie, Finanzwirtschaft und Logistik genannt.

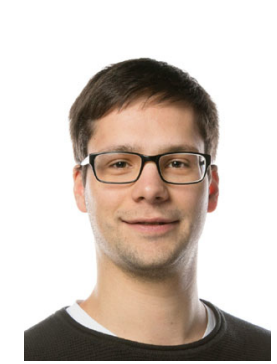

\section{Bernhard Fässler}

ist Assistant Professor an der University of Agder (UiA) in Norwegen und beschäftigt sich mit dem Aufbau eines Kompetenzzentrums für Wiederverwendung und Recycling von Elektrofahrzeugbatterien. Er absolvierte den Bachelorstudiengang Mechatronik an der Fachhochschule Vorarlberg (FHV). Seinen Master in Mechatronik erlangte er im Rahmen eines Double Degree Programs an der FHV und der UiA. Auch das anschließende Doktoratsstudium an der UiA im Bereich Erneuerbare Energien schloss er 2018 in enger Kooperation mit der Fachhochschule Vorarlberg als wissenschaftlicher Mitarbeiter am Forschungszentrum Energie ab. Thematischer Schwerpunkt der Arbeit war die Nutzung autonomer Algorithmen zur Wiederverwendung von gebrauchten Elektrofahrzeugbatterien in stationären Anwendungen.
31. Rheinberger, K., Preissinger, M., Kepplinger, P. (2018): Penalty pricing to control autonomous energy storages for demand side management. 14. Viennese conference on optimal control and dynamic games 2018. Vienna: TU Wien. Invited talk.

32. Samweber, F, Köppl, S., Bogensperger, A., Murmann, A., Zeiselmair, A., Regett, A für Energiewirtschaft, F. (Hrsg.) (2016): Projekt Merit Order Netz-Ausbau 2030 (MONA 2030): Teilbericht Maßnahmenklassifizierung: Endbericht stand. München: FfE Forschungsstelle für Energiewirtschaft e.V., November 2016 edn.

33. Shokrzadeh, S., Bibeau, E. (2016): Sustainable integration of intermittent renewable energy and electrified light-duty transportation through repurposing batteries of plugin electric vehicles. Energy, 106, 701-711.

34. Siano, P. (2014): Demand response and smart grids—a survey. Renew. Sustain. Energy Rev., 30, 461-478.

35. Wayne, M. (1984): Demand planning in the ' 80 s. EPRI J., 9(10), 6.

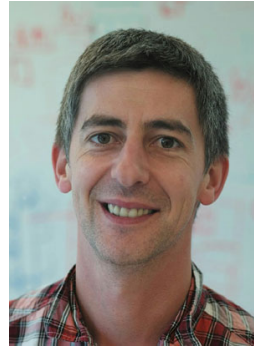

\section{Gerhard Huber}

schloss 2007 das Diplomstudium „Energieund Umweltmanagement" an der Fachhochschule Burgenland ab. Anschließend war er fünf Jahre in der NÖM AG für den operativen Betrieb und die strategische Planung der Energietechnik zuständig. Dazu zählte der tägliche Betrieb der Anlagen sowie die Erneuerung und Erweiterung dieser im Projektmanagement. Seit 2012 ist er als wissenschaftlicher Mitarbeiter am Forschungszentrum Energie an der Fachhochschule Vorarlberg vor Allem für Experimente und Feldversuche zuständig. Dazu zählen die technische Auslegung von Anlagenteilen, die Auswahl der geeigneten Mess- und Regelungstechnik, sowie die Versuchsauswertungen

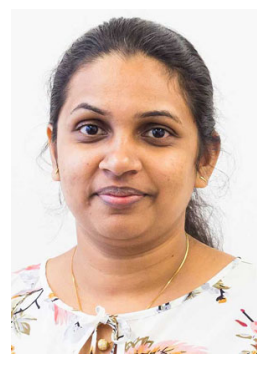

\section{Muhandiram Arachchige Subodha Tha-} rangi Ireshika

hat 2010 ihren Bachelor in Electrical and Information Engineering an der Universität Ruhuna, Sri Lanka, abgeschlossen. 2014 erhielt sie ihren MSc. in Erneuerbare Energien von der University of Agder (UiA) in Norwegen. Anschließend war sie vier Jahre als Assistant Lecturer am Department of Electrical and Information Engineering an der Universität Ruhuna tätig. Ihr Forschungsinteresse gilt dem Demand Side Management elektrischer Fahrzeuge basierend auf unidirektionaler Kommunikation. Seit 2018 ist sie wissenschaftliche Mitarbeiterin in der Forschungsgruppe "Energiesysteme und -komponenten" am Forschungszentrum Energie und im Josef-Ressel Zentrum für Angewandtes Wissenschaftliches Rechnen in Energie, Finanz und Logistik an der Fachhochschule Vorarlberg sowie Doktoratsstudentin an der UiA.

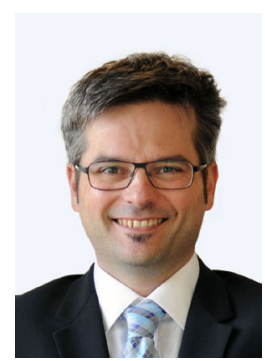

Klaus Rheinberger

schloss 2000 das Diplom- und 2006 das Doktoratsstudium der Physik an der Universität Innsbruck ab. Von 2000 bis 2004 war er wissenschaftlicher Mitarbeiter an der Klinik für Anästhesie und Intensivmedizin, Innsbruck. Seit 2004 ist er wissenschaftlicher Mitarbeiter und Lehrbeauftragter an der Fachhochschule Vorarlberg mit Fokus auf angewandte Mathematik, Data Science und Optimierung in den Bereichen Energie und Finanzwirtschaft. Von 2013 bis 2019 leitete er als Hochschullehrer an der FH Vorarlberg den Masterstudiengang 
Energietechnik und Energiewirtschaft. Seit 2018 ist Klaus Rheinberger als wissenschaftlicher Mitarbeiter in der Forschungsgruppe „Energiesysteme und -komponenten“ am Forschungszentrum Energie und im Josef-Ressel Zentrum für Angewandtes Wissenschaftliches Rechnen in Energie, Finanz und Logistik tätig, wo er seine Expertise in den Bereichen mathematische Optimierung, Finanzwirtschaft und Data Science einbringt.

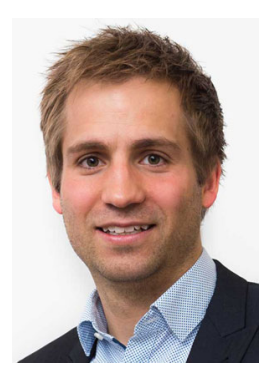

\section{Markus Preißinger}

studierte Umwelt- und Bioingenieurwissenschaften mit Schwerpunkt Energie- und Verfahrenstechnik an der Universität Bayreuth (Deutschland). 2009 nahm er eine Stelle als akademischer Rat auf Zeit am Lehrstuhl für Technische Thermodynamik und Transportprozesse (LTTT) der Universität Bayreuth an. 2012 wurde er Gruppenleiter für Energiesysteme und Energietechnologien am LTTT, zu- dem wurde er im gleichen Jahr zum Geschäftsführer des Zentrums für Energietechnik der Universität Bayreuth ernannt. 2014 promovierte er sich zum Dr.-Ing. mit einer Arbeit über die Stromerzeugung aus industrieller Abwärme. Im August 2017 folgte Markus Preißinger dann dem Ruf der Fachhochschule Vorarlberg auf die illwerke vkw Stiftungsprofessur für Energieeffizienz und leitet seitdem das Forschungszentrum Energie. 